\title{
Avaliação do conhecimento de profissionais de educação física frente à avulsão e fratura dental decorrente da prática esportiva
}

\author{
Assessment of knowledge of physical education professionals regarding dental avulsion and \\ fracture resulting from sports practice \\ Evaluación de los conocimientos de los profesionales de la educación física sobre la avulsión dental \\ y fractura dentaria derivada de la práctica deportiva
}

Recebido: 21/10/2021 | Revisado: 29/10/2021 | Aceito: 05/11/2021 | Publicado: 08/11/2021

\author{
Isadora Caroline Tameirão Gomes \\ ORCID: https://orcid.org/0000-0001-6897-1354 \\ Centro Universitário de Patos de Minas, Brasil \\ E-mail: isadoratameirão@unipam.edu.br \\ Jaíne Joice Amorim \\ ORCID: https://orcid.org/0000-0002-9582-8403 \\ Centro Universitário de Patos de Minas, Brasil \\ E-mail: jaineamorim@unipam.edu.br \\ Paula Guimarães \\ ORCID: https://orcid.org/0000-0002-6290-5898 \\ Centro Universitário de Patos e Minas, Brasil \\ E-mail: paulaguimarães@unipam.edu.br \\ Denise de Souza Matos \\ ORCID: https://orcid.org/0000-0001-6750-6215 \\ Centro Universitário de Patos de Minas, Brasil \\ E-mail: denisesm@unipam.edu.br
}

\begin{abstract}
Resumo
O trauma dental é um problema de saúde pública em decorrência dos danos que podem gerar na criança e adolescente. As lesões provocadas podem ser classificadas quanto à etiologia e consequências provocadas no indivíduo. Os educadores físicos são os profissionais que comumente se deparam com lesões traumáticas, uma vez que os praticantes estão mais expostos a essa situação. Logo, conhecer o manejo correto frente a um dente traumatizado é de extrema importância para o prognóstico do caso. O objetivo desse trabalho é avaliar o conhecimento de profissionais de educação física da Associação de Profissionais de Educação Física (APEF) do município de Patos de Minas frente a um possível caso de avulsão e trauma dental. Para realização dessa pesquisa, 53 profissionais responderam um questionário com perguntas objetivas acerca do tema avulsão dental, por meio da plataforma Google Forms. Ao final do estudo, os profissionais que demonstraram interesse receberam uma devolutiva gratuita acerca do tema abordado. Foi possível observar que a maior parte da amostra é composta por especialistas, com 11 a 20 anos de atuação. Quanto à conduta com um elemento dental avulsionado, apenas 7,5\% dos participantes responderam corretamente, o que pode ser reflexo da grande maioria não ter estudado o assunto durante a sua formação acadêmica, demonstrando um conhecimento insuficiente frente a essa situação.
\end{abstract}

Palavras-chave: Avulsão dentária; Educação física e treinamento; Educadores em saúde.

\begin{abstract}
Dental trauma is a public health problem due to the damage it can cause in children and adolescents. The injuries caused can be classified according to the etiology and consequences caused to the individual. Physical educators are professionals who commonly face traumatic injuries, since practitioners are more exposed to this situation. Therefore, knowing the correct management for a traumatized tooth is extremely important for the prognosis of the case. The objective of this work is to evaluate the knowledge of physical education professionals from the Association of Physical Education Professionals (APEF) in the city of Patos de Minas regarding a possible case of dental avulsion and fracture. To carry out this research, 53 professionals answered a questionnaire with objective questions about the topic of dental avulsion, through the Google Forms platform. At the end of the study, professionals who showed interest received a free feedback on the topic addressed. It was possible to observe that most of the sample is composed of specialists, with 11 to 20 years of experience. As for the conduct with an avulsed dental element, only $7.5 \%$ of the participants answered correctly, which may be a reflection of the vast majority not having studied the subject during their academic training, demonstrating insufficient knowledge regarding this situation.
\end{abstract}

Keywords: Health educators; Physical education and training; Tooth avulsion. 


\section{Resumen}

El trauma dental es un problema de salud pública por el daño que puede causar en niños y adolescentes. Las lesiones causadas pueden clasificarse según la etiología y las consecuencias que le causan al individuo. Los educadores físicos son profesionales que comúnmente enfrentan lesiones traumáticas, ya que los practicantes están más expuestos a esta situación. Por tanto, conocer el manejo correcto de un diente traumatizado es de suma importancia para el pronóstico del caso. El objetivo de este trabajo es evaluar el conocimiento de los profesionales de la educación física de la Asociación de Profesionales de la Educación Física (APEF) de la ciudad de Patos de Minas sobre un posible caso de avulsión dental y fractura dentaria. Para llevar a cabo esta investigación, 53 profesionales respondieron un cuestionario con preguntas objetivas sobre el tema de la avulsión dental, a través de la plataforma Google Forms. Al final del estudio, los profesionales que mostraron interés recibieron una retroalimentación gratuita sobre el tema abordado. Se pudo observar que la mayor parte de la muestra está compuesta por especialistas, con 11 a 20 años de experiencia. En cuanto a la conducta con un elemento dental avulsionado, solo el 7,5\% de los participantes respondió correctamente, lo que puede ser reflejo de que la gran mayoría no ha estudiado el tema durante su formación académica, demostrando un conocimiento insuficiente sobre esta situación.

Palabras clave: Avulsión de diente; Educación y entrenamiento físico; Educadores en salud.

\section{Introdução}

O traumatismo dentário é um problema de saúde pública, devido a sua alta prevalência e danos gerados ao indivíduo (Mesquita et al, 2017). As lesões traumáticas decorrentes desse evento são um dos principais motivos da procura de serviços odontológicos de urgência por parte de crianças e adolescentes, os quais são grupos comumente afetados (Rodrigues et al., 2021). As etiologias desses impactos são diversas: quedas, acidentes de trânsito, acidente de bicicleta, violência, acidente doméstico, acidente de trabalho e acidente pela prática esportiva (Costa et al.,2014).

No que se refere à epidemiologia, na dentição decídua os traumas são mais comuns entre 1 e 3 anos, momento no qual as crianças começam a explorar ambientes. Os traumas afetando dentes permanentes acontecem mais comumente em crianças de 7 a 12 anos, as quais ainda apresentam desenvolvimento radicular incompleto em alguns elementos e periodonto resiliente e, em seguida, os jovens de 16 a 20 anos (Menegotto, Scatena, Pereira, Werle \& Oliveira, 2017). No geral, os últimos dois grupos supracitados são os mais acometidos pelo fato de praticarem esportes que oferecem um maior risco (Lopes \& Ferreira, 2017). Os dentes mais afetados são os superiores anteriores para ambas as dentições. Quanto ao gênero, estudos epidemiológicos demonstram que os homens tendem a serem mais atingidos quando comparado às mulheres (Traebert, Marcon \& Lacerda, 2010, Marinho, Manso, Colares \& de Andrade, 2013) e os fatores predisponentes aos traumas incluem overjet acentuado, protrusão maxilar e falta de proteção labial (Forsberg \& Tedestam, 1993; Traebert et al, 2003).

Para Andreasen (2000), o trauma dental classifica-se em: lesões aos tecidos duros dentários e da polpa e lesões aos tecidos de sustentação (luxações). Pode haver uma simples fratura de esmalte a uma completa avulsão do elemento dental e o tratamento correto e de urgência nessas situações faz toda diferença para um prognóstico favorável do caso (Costa et al., 2014). Sendo assim, o conhecimento sobre as medidas de urgência frente a essa situação, se faz necessário para os diversos grupos de profissionais que acompanham praticantes de esportes, dentre eles, os profissionais da educação física (Levin, Samorodnitzky, Schwartz-Arad, \& Geiger 2007).

Os educadores físicos, possuem a função de estimular a prática esportiva, bem como lidar com as diversas situações que podem advir decorrentes desses atos, já que, há exposição dos praticantes ao risco de certas lesões (Antunes, Souza, Gonçalves, Crespo \& Antunes, 2016). Diante de avulsão do elemento dental permanente, a conduta mais adequada por parte desse profissional, de acordo com as diretrizes da International Dental Traumatology Association (2020) seria reimplantá-lo no alvéolo imediatamente e, caso não seja possível, deve-se imergir o dente em meio próprio como leite, saliva ou solução salina para garantir a viabilidade das células do ligamento periodontal e encaminhar o paciente ao dentista o mais rápido possível, sendo que medidas como esta fazem toda diferença para o sucesso e tratamento do caso (Andersson et al., 2012).

Conhecer o nível de informação por parte desses profissionais, bem como orientá-los quanto às condutas corretas frente a um traumatismo dentário é de extrema importância para minimizar as perdas dentárias que podem interferir em 
questões estéticas, na função e podem ainda gerar problemas psicológicos oriundos de traumas dentais decorrentes da prática esportiva (Mesquita et al., 2017), uma vez que o profissional de educação física pode ser o responsável pelos primeiros cuidados e primeiro atendimento prestado ao paciente (Granville-garcia, Santos \& Menezes,2007).

O presente trabalho se justifica devido à relevância da abordagem do tema junto aos profissionais que atuam na orientação da prática de esportes, uma vez que a prevalência dos traumas durante os exercícios tem se mostrado crescente, em função do aumento da prática de esportes que envolve intenso contato com os demais participantes. Por fim, são necessárias pesquisas nesse âmbito, uma vez que existe uma escassez de trabalhos com profissionais atuantes na área.

Portanto, levando-se em consideração que, de acordo com a literatura, uma das principais causas dos traumatismos dentários advém da prática esportiva, o presente trabalho se propõe a avaliar o nível de conhecimento de profissionais de educação física da Associação de Profissionais de Educação Física (APEF), no município de Patos de Minas, frente a um possível caso de avulsão e trauma dental.

\section{Metodologia}

\subsection{Aspectos éticos}

Inicialmente, o projeto de pesquisa foi submetido e aprovado pelo Comitê de Ética em Pesquisa do Centro Universitário de Patos de Minas - UNIPAM, sob parecer número 4.652.123 e CAAE 44099321.9.0000.5549 estando de acordo com a Resolução 196/96 do Conselho Nacional de Saúde. Todos os participantes assinaram o Termo de Consentimento Livre e Esclarecido consentindo sua participação na pesquisa.

\subsection{Delineamento do estudo e recrutamento da amostra}

Trata-se de um estudo transversal de caráter quantitativo e descritivo que foi realizado por meio de amostragem por conveniência, na qual fizeram parte da pesquisa, 53 graduados em educação física da Associação de Profissionais de Educação Física (APEF) do município de Patos de Minas. Tais profissionais foram convidados a responder um questionário abordando temas relativos ao traumatismo dental, composto por 13 perguntas objetivas de múltipla escolha. O questionário continha 3 partes, sendo a primeira com perguntas para caracterizar a amostra, a segunda com questionamentos relacionados a experiência profissional e a terceira, acerca de condutas específicas frente a um trauma propriamente dito, e em especial a avulsão.

O período de coleta de dados foi do dia 01/05/2021 ao dia 31/05/2021. Em decorrência da pandemia pelo Sars-CoV-2 os questionários foram realizados por meio do aplicativo de gerenciamento de pesquisa do Google, o Google Forms, e enviados via WhatsApp, levando em conta que o termo de consentimento livre e esclarecido (TCLE) foi apresentado previamente ao preenchimento do formulário com a opção de recusa e aceite na participação da pesquisa, para que o trabalho fosse realmente voluntário e para que todos os participantes entendessem a natureza e o objetivo do estudo.

No que se refere aos procedimentos que envolvem contato por meio virtual com os possíveis participantes da pesquisa, foi gerado um link de acesso ao questionário no qual cada profissional de educação física obteve entrada individualmente, portanto sem a necessidade de listas que identificassem os demais convidados e seus dados de contato. Além disso, os participantes tiveram o direito de não responder a qualquer questão, não necessitando de justificativas ou explicações para tal e o mesmo foi instruído no TCLE ser recomendável guardar uma cópia do documento eletrônico em seus arquivos. O profissional convidado teve acesso previamente ao tema da pesquisa para que pudesse decidir se desejava mesmo participar do estudo.

Com relação à segurança na transferência e no armazenamento de dados, ficou sob responsabilidade do pesquisador o armazenamento, confidencialidade e sigilo das informações coletados. Ao final da obtenção de dados, todos os materiais foram 
transferidos para outro dispositivo local e os aspectos que se encontravam na plataforma virtual Google Forms foram apagados para evitar qualquer intercorrência. Por fim, quanto ao conteúdo dos dados tramitados, no TCLE constavam todas as explicações necessárias para que o participante se sentisse o mais seguro possível e as regras e direitos do mesmo foram explícitas nesse documento. Ademais caso o integrante desistisse da participação, os pesquisadores se comprometeram a enviar uma resposta de ciência desse evento, retirando os dados do mesmo do estudo e, além disso, essas informações também se encontram no TCLE, bem como a forma com que o consentimento foi obtido para realização da presente pesquisa.

\subsection{Critérios de inclusão e exclusão}

Foram incluídos na pesquisa os profissionais de educação física que atuam no município de Patos de Minas, que fazem parte da APEF, com graduação completa em educação física e atuantes na área.

Foram excluídos os participantes que não apresentam familiaridade com o preenchimento do formulário online, aqueles que não concordaram com a pesquisa e não assinaram o termo de consentimento livre e esclarecido.

\subsection{Análise de riscos e benefícios}

O presente estudo apresentou classificação de risco de grau mínimo para os participantes, sendo eles: a possibilidade da quebra do sigilo e anonimato, bem como o constrangimento ao responder as perguntas.

A fim de minimizar esses riscos, a pesquisa foi voluntária, os adeptos foram identificados apenas com as iniciais, o questionário, por ser enviado via WhatsApp, garantiu que o profissional respondesse no momento que considerasse mais oportuno, preservando assim sua privacidade e, por fim, os pesquisadores asseguraram a confidencialidade dos dados e respostas individuais obtidos com a pesquisa. Além disso, os pesquisadores buscaram se inteirar sobre a política de privacidade da plataforma Google Forms, a transferir para outro dispositivo os dados coletados, excluindo todas as informações que se encontravam na base de dados utilizada na realização da pesquisa. Por fim, o TCLE apresentou explicações como: regras e direitos do participante, a forma que o consentimento foi obtido, o comprometimento por parte dos pesquisadores de retirarem os dados de algum profissional que por ventura desistiu da participação da pesquisa, e o envio de uma resposta de ciência acerca desse evento e da retirada de suas informações do estudo.

No que se refere aos benefícios, um deles seria a possibilidade dos profissionais se atentarem quanto à importância do tema abordado no questionário, uma vez que este pode passar despercebido durante a graduação. Ao final do preenchimento do questionário o participante foi indagado se desejava receber um informativo sobre as condutas corretas de urgência frente a avulsão do elemento dental, caso a resposta fosse positiva, o informativo seria enviado para o e-mail do participante de forma gratuita, proporcionando conhecimento acerca de primeiros socorros relacionados aos traumatismos buco-dentários.

\subsection{Análise estatística}

Para categorizar o grupo em estudo, foram realizadas as estatísticas descritivas, com tabelas de frequência para as variáveis qualitativas. O teste de hipótese não paramétrico $\mathrm{G}$ para independência foi utilizado para estimar a associação entre as diferentes categorias dos dados. Em todo o estudo, estipulou-se o nível de significância em 5\%, sendo as análises realizadas através do software Bioestat versão 5.3.

Foi realizado também teste não-paramétrico para duas amostras independentes, semelhante em todos os seus aspectos ao do Qui-Quadrado, para dados categóricos. Os escores foram mensurados a nível nominal ou ordinal, e as amostras poderiam 
apresentar duas ou mais categorias dispostas em tabelas de contingência 1 x c. Os graus de liberdade foram assim calculados: (1 $-1) \times(c-1)$.

\section{Resultados}

Foram convidados a participar do estudo 119 profissionais vinculados à APEF do município de Patos de Minas, entretanto, ao final do prazo estipulado para respostas ao questionário on-line foram obtidas 55 participações das quais 2 foram excluídas por não terem respondido o questionário por completo. Foi perguntado, inicialmente, para caracterização da amostra, o grau de formação, o tempo de trabalho como profissional de educação física e o local de atuação. Os dados referentes à parte 1 do questionário com as informações que caracterizam a amostra estão descritos na tabela 1.

Tabela 1 - Caracterização da amostra.

\begin{tabular}{|c|c|c|c|}
\hline $\begin{array}{l}\text { Descrição dos parâmetros } \\
\text { analisados }\end{array}$ & $\begin{array}{c}\text { Classes dos parâmetros } \\
\text { analisados }\end{array}$ & $\begin{array}{c}\text { Tamanho da } \\
\text { amostra }\end{array}$ & $\begin{array}{c}\text { Frequência } \% \\
\text { (n) }\end{array}$ \\
\hline \multirow{4}{*}{ Grau de formação } & Graduação & \multirow{4}{*}{53} & $41,5(22)$ \\
\hline & Especialização & & $45,3(24)$ \\
\hline & Mestrado & & $11,3(6)$ \\
\hline & Doutorado & & $1,9(1)$ \\
\hline \multirow{3}{*}{ Tempo de trabalho } & Menos que 10 anos & \multirow{3}{*}{53} & $34(18)$ \\
\hline & Entre 11 e 20 anos & & $45,2(24)$ \\
\hline & Mais que 20 anos & & $20,8(11)$ \\
\hline \multirow{4}{*}{ Local de atuação } & Academia e/ ou clube & \multirow{4}{*}{63} & $50,8(32)$ \\
\hline & Escola & & $33,3(21)$ \\
\hline & Faculdade & & $4,8(3)$ \\
\hline & Personal & & $11,1(7)$ \\
\hline
\end{tabular}

Fonte: Dados da pesquisa (2021).

As partes 2 e 3 do questionário contaram com 10 perguntas referentes à conduta dos profissionais de educação física frente a um trauma dental decorrente da prática esportiva, em especial a avulsão. A frequência das respostas frente aos parâmetros analisados está descrita na tabela 2. 
Tabela 2 - Frequência das classes dos parâmetros analisados.

\begin{tabular}{|c|c|c|c|}
\hline Descrição dos parâmetros analisados & Classes dos parâmetros analisados & $\begin{array}{l}\text { Tamanho da } \\
\text { amostra }\end{array}$ & Frequência \% (n) \\
\hline \multirow{2}{*}{$\begin{array}{l}\text { Durante a formação teve alguma disciplina } \\
\text { que abordou o tema trauma dental? }\end{array}$} & Sim & \multirow{2}{*}{53} & $5,7(3)$ \\
\hline & Não & & $94,3(50)$ \\
\hline \multirow{2}{*}{$\begin{array}{l}\text { Presenciou uma situação em que houve } \\
\text { trauma dos elementos dentários? }\end{array}$} & Sim & \multirow{2}{*}{53} & $50,9(27)$ \\
\hline & Não & & $49,1(26)$ \\
\hline \multirow{2}{*}{$\begin{array}{l}\text { Em alguma dessas situações, em que } \\
\text { presenciou trauma dos elementos dentário o } \\
\text { dente saiu todo para fora da boca (avulsão)? }\end{array}$} & Sim & \multirow{2}{*}{27} & $3,7(1)$ \\
\hline & Não & & $96,3(26)$ \\
\hline \multirow{4}{*}{$\begin{array}{l}\text { Qual a melhor conduta a ser adotada quando } \\
\text { um dente permanente, em uma situação de } \\
\text { trauma sair totalmente para fora da boca } \\
\text { (avulsão)? }\end{array}$} & $\begin{array}{l}\text { Colocar em um recipiente contendo } \\
\text { líquido e encaminhar ao dentista }\end{array}$ & \multirow{4}{*}{53} & $45,3(45)$ \\
\hline & $\begin{array}{l}\text { Embrulhar o dente em um papel } \\
\text { toalha e encaminhar ao serviço } \\
\text { odontológico/médico de urgência }\end{array}$ & & $47,2(25)$ \\
\hline & $\begin{array}{c}\text { Reimplantá-lo no local o mais rápido } \\
\text { possível }\end{array}$ & & $7,5(4)$ \\
\hline & $\begin{array}{l}\text { Descartar o dente em lixo correto } \\
\text { para evitar contaminações }\end{array}$ & & $0,0(0)$ \\
\hline \multirow{5}{*}{$\begin{array}{l}\text { Por quanto tempo um dente pode permanecer } \\
\text { fora da boca garantindo posteriormente um } \\
\text { prognóstico favorável do caso? }\end{array}$} & 15 minutos & \multirow{5}{*}{53} & $7,5(4)$ \\
\hline & 30 minutos & & $28,3(15)$ \\
\hline & 1 hora & & $37,7(20)$ \\
\hline & 2 horas & & $15,2(8)$ \\
\hline & Sem limite de tempo & & $11,3(6)$ \\
\hline \multirow{5}{*}{$\begin{array}{l}\text { Qual o meio mais indicado para armazenar } \\
\text { um dente avulsionado (quando o dente sai } \\
\text { todo para fora da boca)? }\end{array}$} & Água filtrada & \multirow{5}{*}{53} & $28,3(15)$ \\
\hline & Leite & & $11,3(6)$ \\
\hline & Saliva & & $7,5(4)$ \\
\hline & Soro Fisiológico & & $52,9(28)$ \\
\hline & Água de côco & & $0,0(0)$ \\
\hline \multirow{2}{*}{$\begin{array}{l}\text { Para qual profissional devo encaminhar o } \\
\text { paciente em casos de trauma dental? }\end{array}$} & Cirurgião-dentista & \multirow{2}{*}{53} & $96,2(51)$ \\
\hline & Médico & & $3,8(2)$ \\
\hline \multirow{2}{*}{$\begin{array}{l}\text { Por qual parte/porção deve-se pegar o dente } \\
\text { em caso de avulsão (quando o dente sai todo } \\
\text { para fora da boca)? }\end{array}$} & Coroa & \multirow{2}{*}{53} & $98,1(52)$ \\
\hline & Raiz & & $1,9(1)$ \\
\hline \multirow{2}{*}{$\begin{array}{l}\text { Você acredita que um trauma na dentição } \\
\text { decídua (de leite) pode afetar a dentição } \\
\text { permanente? }\end{array}$} & Sim & \multirow{2}{*}{53} & $86,8(46)$ \\
\hline & Não & & $13,2(7)$ \\
\hline \multirow{3}{*}{$\begin{array}{l}\text { Qual a melhor conduta a ser adotada quando } \\
\text { um aluno fratura um dente e te mostra o } \\
\text { fragmento dental? }\end{array}$} & $\begin{array}{c}\text { Descartar o fragmento em lixo } \\
\text { apropriado. }\end{array}$ & \multirow{3}{*}{53} & $0,0(0)$ \\
\hline & $\begin{array}{l}\text { Encaminhar o aluno ao dentista com } \\
\text { o fragmento para o mesmo analisar a } \\
\text { melhor conduta. }\end{array}$ & & $100(53)$ \\
\hline & $\begin{array}{l}\text { Tentar colar o fragmento o mais } \\
\text { rápido possível }\end{array}$ & & $0,0(0)$ \\
\hline
\end{tabular}

Fonte: Dados da pesquisa (2021). 
Após análise dos resultados foi possível observar que a abordagem do tema "trauma dental" durante a formação do profissional de educação física, de acordo com a amostra analisada, ainda é pouco comum, uma vez que apenas 5,7\% dos profissionais relataram terem tido informações sobre o assunto.

Durante o preenchimento do questionário os profissionais participantes da pesquisa foram indagados sobre condutas e situações relativas ao trauma dental que são possíveis de acontecer durante a prática esportiva. Cada pergunta continha alternativas que foram consideradas corretas ou mais indicadas de acordo com as recomendações da International Association of Dental Traumatology (IADT, 2020). Tais alternativas foram realçadas na tabela 2. Após análise dos resultados foi possível observar que muitos profissionais assinalaram respostas consideradas incorretas, o que pode ser justificado pelo fato da maioria não terem tido informações sobre o assunto em sua formação.

Após realização do teste de independência foi possível constatar que não houve associação entre o grau de formação dos entrevistados e o fato de terem tido alguma disciplina onde foi abordado o tema trauma dental $(p=0,1739)$. Outras associações que não foram encontradas foram com relação ao tempo de trabalho como educador físico versus presenciar uma situação em que houve trauma dos elementos dentários $(p=0,4408)$ e o local de atuação versus presenciar uma situação em que houve trauma dos elementos dentários $(p=0,4593)$.

Foram analisadas as associações entre outras variáveis com o fato de o entrevistado ter tido informações sobre o tema "trauma dental" durante a sua formação, das quais não foram encontradas relação, tais como: 1- Melhor conduta a ser adotada quando um dente permanente, em uma situação de trauma sair totalmente para fora da boca (avulsão) versus ter disciplina que abordou o tema trauma dental $(p=0,1878) ; 2$ - Escolha do meio mais indicado para armazenar um dente avulsionado (quando o dente sai todo para fora da boca) versus ter disciplina que abordou o tema trauma dental ( $p=0,2102)$; 3- Profissional ao qual deve-se encaminhar o paciente em casos de trauma dental versus ter disciplina que abordou o tema trauma dental $(p=0,6259)$; 4- Parte/porção que se deve pegar o dente em caso de avulsão versus ter disciplina que abordou o tema trauma dental $(p=$ 0,7316); 5- Acreditar que um trauma na dentição decídua (de leite) pode afetar a dentição permanente versus ter disciplina que abordou o tema trauma dental $(p=0,3491)$.

A única associação encontrada foi com relação à variável "tempo que um dente pode permanecer fora da boca garantindo posteriormente um prognóstico favorável do caso" versus "ter disciplina que abordou o tema trauma dental". Para essa associação foi observada que ter disciplina que abordou o tema trauma dental influenciou na resposta $(p=0,0378)$. Nesta associação, profissionais que possuem a disciplina "trauma dental" no currículo afirmaram que o prazo máximo que um dente pode permanecer fora da boca garantindo posteriormente um prognóstico favorável do caso é de até 30 minutos, entretanto, de acordo com a IADT (2020), o mais indicado seria 15 minutos com prognóstico favorável, e 30 minutos podendo ou não ser favorável, uma vez que as células podem já estar comprometidas.

\section{Discussão}

Sabe-se que a prática esportiva é responsável pela maioria das lesões, uma vez que esportes como rugby, judô ou a versão americana do futebol, entre outros, são de intenso contato, envolvendo agarrar, tocar e atacar os participantes da prática (Lam, 2016). De acordo com pesquisas realizadas pela American Dental Association, mais de 5 milhões de dentes são avulsionados por ano, e a prática esportiva é responsável por até 39\% dessas ocorrências (Santos, 2018). Tal acontecimento se dá por causa do aumento da competitividade e intensidade da prática entre os esportistas, bem como a velocidade do jogo que aumenta o potencial de um eventual traumatismo (Ferrari, Simi \& Medeiros, 2001).

O manejo de dentes traumatizados é de extrema importância para o prognóstico do caso. É necessário que treinadores e profissionais de educação física, que têm contato direto com os alunos praticantes de esporte, estejam aptos a providenciar cuidado imediato em casos de trauma dental (Chan, Wong \& Cheung, 2001). Inicialmente, quanto a caracterização da amostra 
na presente pesquisa, verificou-se que dentre os participantes $(n=53)$ a maior parte é composta de especialistas, e relatam ter entre 11 e 20 anos de atuação como profissional de educação física e, além disso, mais da metade afirmam que atuam em academia e/ou clube.

No que se refere à conduta dos profissionais frente a um trauma, destaca-se que quase todos os participantes relataram não ter contato com o assunto durante sua formação acadêmica, corroborando com o fato apontado por diversos autores de que a maioria das instituições de ensino superior que oferecem a formação em Educação Física não possuem em sua grade curricular disciplinas que abordem o assunto traumatismo dentário (Pacheco et al., 2003, Holan, Cohenca, Brin \& Sgan-Cohen, 2006, Jorge et al., 2009). Diante disso, percebe-se uma lacuna de conhecimento científico com relação ao conteúdo abordado, congruente ao estudo de Silva et al. (2013), no qual 64,3\% dos alunos entrevistados de um centro universitário demonstraram não terem tido contato com o tema até então, demonstrando conhecimento insuficiente para lidar com essa situação, expondo a necessidade de primeiros socorros buco-dentários serem inseridos ainda na graduação.

Além disso, os participantes desta pesquisa foram indagados se já presenciaram alguma situação de trauma e mais da metade afirmaram terem presenciado, dos quais apenas a minoria presenciou o dente sair totalmente para fora da boca, ou seja, avulsão do elemento dental, o que está de acordo com os estudos encontrados na literatura, como o de Santos, Silva, Cardoso e Xavier (2017) em que apenas 14,3\% dos participantes presenciaram casos de avulsão. Partindo desse pressuposto, uma associação que não foi encontrada no estudo, refere-se ao tempo como educador físico versus presenciar uma situação de trauma, corroborando com o estudo de Granville-Garcia et al. (2007), no qual os autores também afirmam que a experiência profissional ou o grau de formação pareceu não influenciar na escolha das respostas corretas ou presenciar situações de trauma.

Quando questionados sobre qual a melhor conduta a ser adotada quando um dente permanente em uma situação de trauma sair totalmente para fora da boca (avulsão), quase metade da amostra afirmou que a melhor conduta seria embrulhar o dente em um papel toalha e encaminhar ao serviço odontológico/médico de urgência, seguido por colocar em um recipiente contendo líquido e encaminhar ao dentista, ao passo que a minoria respondeu reimplantar o dente, o mais rápido possível. Para Ceallaigh, Ekanaykaee, Beirne e Patton (2006), a melhor conduta em casos de avulsão de dentes permanentes deve ser reimplantar o dente no local de origem imediatamente após o trauma. Entretanto nos casos em que o profissional responsável pela prática esportiva não se sentir apto a realizar este procedimento, não se deve transportar o dente seco, como embrulhado em um papel toalha, pelo fato de ressecar as células do ligamento periodontal que permanecem na raiz, podendo necrosá-las o que pode levar a um prognóstico desfavorável do dente reimplantado (Andreasen, 2007).

Ao responderem sobre quanto tempo um dente pode permanecer fora da boca garantindo posteriormente um prognóstico favorável do caso, a maioria respondeu 1 hora enquanto que a menor parte dos entrevistados respondeu 15 minutos. De acordo com Fouad et al. (2020) a desidratação da superfície radicular acontece em questão de minutos e, portanto, em um dente permanente avulsionado com cerca de 60 minutos fora da boca, as células estarão provavelmente inviáveis, gerando um prognóstico duvidoso do caso, entretanto, caso o tempo fora da boca seja inferior a 60 minutos, porém conservado em meio apropriado, as chances de salvar o dente aumentam, podendo as células estarem comprometidas, mas ainda viáveis. Logo, se o dente for reimplantado no local imediatamente, ou dentro de 15 minutos, ou ainda conservado em meio adequado, dentro de 30 minutos, as células estarão provavelmente viáveis, garantindo uma maior sobrevida do dente (Adreasen \& Andreasen, 1995).

Os profissionais, possuem um conhecimento inadequado a respeito do meio mais indicado para armazenar um dente avulsionado, uma vez que mais da metade afirmou ser o soro fisiológico o meio mais propício. O armazenamento também requer cuidado. De acordo com o guia "Avulsão de dentes permanentes" (Fouad et al. 2020), a ordem decrescente de preferência para o transporte é: leite, solução balanceada de Hank (HBSS), saliva (após cuspir em um copo, por exemplo) e solução salina, sendo todos eles meios de armazenamento adequados e convenientes, assim como a água de coco, a qual não 
foi marcada por nenhum participante, entretanto, esse líquido apresenta nutrientes que são importantes para preservação da viabilidade das células (Poi et al., 2013). Os meios indicados podem ser todos os citados, em especial o leite, segundo item, menos citado. Para Adnan et al. (2018) a água é um meio pobre, contudo é preferível ao transporte seco do dente, contrapondo assim com Andreasen e Andreasen (1995) que afirmaram que o armazenamento em água de torneira por mais de 20 minutos pode levar a reabsorção radicular, bem como soluções esterilizantes (álcool) ou soluções não fisiológicas como solução salina caseira.

Nota-se ainda que a maioria não escolheria o leite, que consiste em um meio adequado em virtude das suas propriedades fisiológicas, incluindo $\mathrm{pH}$ e osmolaridade compatíveis com as células do ligamento periodontal, além de estar disponível facilmente nos locais onde ocorrem os acidentes e por estarem livre de microrganismos, quando bem acondicionados. Esta substância tem sido indicada também pela American Association of Endodontists (2004), por manter a viabilidade das células do ligamento periodontal (Blomlof, Lindskog, Andersson, Hedström \& Hammarström 1983; Beofor et al., 1985; Gomes et al., 2009).

O consultório odontológico foi considerado o local de escolha para encaminhar o aluno após uma situação de trauma , evidenciando o conhecimento dos profissionais de educação física frente à necessidade de acompanhamento por um profissional capacitado para, desta forma, permitir um plano de tratamento adequado ao paciente, corroborando com o estudo de Granville-Garcia et al. (2007), o qual também mostra educadores físicos cientes do profissional mais indicado para o tratamento do traumatismo dento alveolar. Entretanto, é importante salientar que qualquer pessoa devidamente informada e preparada pode realizar, em caso de avulsão de dente permanente, o reimplante dentário (Westphalen et al., 2007).

Em relação a qual parte/porção do dente deve-se manusear em caso de avulsão, foi praticamente unânime a resposta coroa do dente. De acordo com Côrtes e Bastos, (2004), uma grande parte do sucesso de um reimplante depende da manipulação do dente no momento da avulsão. Portanto, deve-se evitar tocar ou raspar a raiz, para evitar danos às células do ligamento periodontal, necrose e posterior desenvolvimento de reabsorção radicular, após o reimplante do elemento, sendo, portanto, o mais indicado manusear o dente apenas por sua coroa, evitando-se qualquer contato com a raiz do elemento (Fouad et al., 2020).

Verificou-se que a maioria dos profissionais, acreditam que um trauma na dentição decídua pode afetar a dentição permanente. Para Day et al. (2020) há uma estreita relação entre o ápice do dente decíduo e o germe do permanente e, portanto, em um caso de trauma na primeira dentição, as consequências também podem se manifestar na segunda dentição. Pode haver: malformação dentária, dentes retidos, distúrbios de erupção (Gondim et al., 2011) hipoplasia, alteração de cor e até mesmo casos mais graves como deslocamentos do elemento dentário ou ainda a paralização da formação do germe permanente (Wanderley, Coutinho \& Lage-Marques 2013).

No presente estudo houve uma resposta uniforme onde ao serem questionados sobre o que fazer quando um aluno sofre um trauma com fratura do elemento dental e tem o pedaço do dente fraturado em mãos. Toda a amostra $(\mathrm{n}=53)$ respondeu que encaminharia o aluno ao dentista com o fragmento dental, não tentando colar ou até mesmo descartar o fragmento do dente no lixo. Para Bourguignon et al. (2020) o tratamento de escolha nesse caso pode ser alternativo e o dentista deve avaliar a melhor conduta a ser adotada, podendo optar por realizar a colagem do fragmento ou uma restauração em resina composta avaliando, de acordo com a extensão da fratura, se será ou não necessário uma proteção pulpar, ou ainda, dependendo da extensão e localização da fratura apenas alisar as bordas do elemento dental fraturado para evitar lacerações nos tecidos moles adjacentes (Losso, Tavares, Bertoli \& Baratto, 2011)

Por fim, pode-se observar com o presente estudo que não houve associação entre o local de atuação do profissional versus presenciar uma situação em que houve trauma dos elementos dentários, contrapondo com os estudos de Alves, Freitas, Rosendo, Gominho, e Sarmento, (2015) no qual os autores acreditam que há uma incidência maior de traumas em ambiente 
escolar, tendo em vista que a criança passa grande parte do tempo nesse ambiente. Os autores afirmam que as atividades recreativas praticadas nesse local são fatores que predispõe o traumatismo, devendo-se, portanto, ser alvo de vigilância constante por parte dos profissionais de educação que deverão ser habilitados na prestação dos primeiros socorros.

A única associação encontrada na pesquisa refere-se a ter estudado o assunto trauma dental durante a formação acadêmica do entrevistado versus tempo em que o dente pode permanecer fora da boca, no qual a maioria dos participantes responderam o tempo de 30 minutos. Para a International Association of Dental Traumatology (2020) o tempo limite para manter as células do ligamento periodontal viáveis, garantindo prognóstico favorável e minimizando eventuais sequelas ao dente,), é de 15 minutos, entretanto caso bem conservado com 30 minutos extra alveolar, o dente ainda pode apresentar sucesso ao realizar o reimplante.

Logo, é nítido que o gerenciamento de um trauma envolvendo tecidos moles e duros da boca deve começar no momento em que ocorreu a lesão, a fim de evitar que as primeiras condutas sejam realizadas apenas pelo cirurgião-dentista tardiamente, o que pode afetar a recuperação da criança e do adolescente que sofreu o acidente. Isso expõe a necessidade de educar os profissionais de educação física para um manejo correto do aluno no momento imediato pós-trauma (Diangelis et al., 2012; Lam, 2016).

\section{Conclusão}

Diante do exposto, percebe-se que os profissionais de educação física, possuem um conhecimento científico limitado a respeito do tema traumatismo dental, uma vez que demonstram condutas inapropriadas nos casos de avulsão que podem ocorrer. Esse despreparo é reflexo da abordagem do tema durante a formação acadêmica dos alunos de educação física ser pouco comum, suscetibilizando sequelas e um pior prognóstico dos casos de traumatismo.

\section{Referências}

Adnan, S. et al (2018). Qual o meio mais recomendado para o armazenamento e transporte de dentes avulsionados? Uma revisão sistemática. Dent Traumatol, 34(1), 59-70.

Alves, L. S. B., Freitas, V. J. G., Rosendo, R. A., Gominho, L. F., \& de AP Sarmento, T. C. (2015). Avaliação do conhecimento de professores do ensino fundamental da rede particular sobre atendimento imediato de vítima de traumatismo dental. Revista da Faculdade de Odontologia-UPF, 20 (3), $302-307$.

Endodontia, A. A. (2004). Recommended guidelines of the American Association of Endodontists for Traumatic Dental Injuries.

Andreasen, JO.; Andreasen, FM (1995). Livro didático e Atlas colorido de lesões traumáticas dos dentes. Copenhagen: Munksgaard.

Andreasen, J. O., Borum, M. K., Jacobsen, H. L., \& Andreasen, F. M. (1995). Replantation of 400 avulsed permanent incisors. 4 . Factors related to periodontal ligament healing. Dental traumatology, 11(2), 76-89.

Andreasen, J. O., Andreasen, F. M., Bakland, L. K., \& Flores, M. T. (2000). Manual de traumatismo dental. Artmed.

Andersson, L. et al (2012). Diretrizes da International Association of Dental Traumatology para o tratamento de lesões dentárias traumáticas: Avulsão dos dentes permanentes. Rev.Dental Traumatol, 28,( 2),88-96.

Andreasen, J. O., Andreasen, F. M., \& Andersson, L. (Eds.). (2018). Textbook and color atlas of traumatic injuries to the teeth. John Wiley \& Sons.

Antunes, L. A. A., Souza, H. M. R. D., Gonçalves, P. H. P. D. Q., Crespo, M. A., \& Antunes, L. S. (2016). Trauma dental e protetor bucal: conhecimento e atitudes em estudantes de graduação em Educação Física. Revista Brasileira de Educação Física e Esporte, 30, $287-294$.

Belford, D. A., Rogers, M. L., Regester, G. O., Francis, G. L., Smithers, G. W., Liepe, I. J., ... \& Ballard, F. J. (1995). Milk-derived growth factors as serum supplements for the growth of fibroblast and epithelial cells. In Vitro Cellular \& Developmental Biology-Animal, 31(10), 752-760.

Blomlöf, L., Lindskog, S., Andersson, L., Hedström, K. G., \& Hammarström, L. (1983). Storage of experimentally avulsed teeth in milk prior to replantation. Journal of Dental Research, 62(8), 912-916.

Bourguignon, C., Cohenca, N., Lauridsen, E., Flores, M.T., Connell, A. O., Day, P. ... Levin, L. (2020). Diretrizes da International Assoctiation of Dental Traumatology para o tratamento de lesões dentárias traumáticas: Fraturas e luxações. Rev.Dental Traumatology, 36(4), 314-330. 
Ceallaigh, P. Ó., Ekanaykaee, K., Beirne, C. J., \& Patton, D. W. (2007). Diagnosis and management of common maxillofacial injuries in the emergency department. Part 5: Dentoalveolar injuries. Emergency Medicine Journal, 24(6), 429-430.

Chan, A. W., Wong, T. K., \& Cheung, G. S. (2001). Lay knowledge of physical education teachers about the emergency management of dental trauma in Hong Kong. Dental Traumatology, 17(2), 77-85.

Côrtes, M. I. S., \& Bastos, J. V. (2004). Lesões traumáticas na dentição permanente. Estrela C. Ciência endodôntica. São Paulo: Artes Médicas, 799-918.

Costa, L. E. D., Queiroz, F. D. S., Nóbrega, C. B. C., Leite, M. S., Nóbrega, W. F. S., \& Almeida, E. R. D. (2014). Trauma dentário na infância: avaliação da conduta dos educadores de creches públicas de Patos-PB. Revista de Odontologia da UNESP, 43, 402-408.

Day, P. F., Flores, M. T., Connell, A.O., Abott, P. V., Tsilingaridis, G., Fouad A. F. ...Levin,L. (2020). International Association of Dental Traumatology guidelines for the management of traumatic dental injuries: Injuries in the primary dentition. Dental Traumatology, 36, (4), 343-359.

Diangelis, A.J., Andreasen, J.O., Ebeleseder, K.A., Kenny, D.J., Trope, M., Sigurdsson, A, ... Tsukiboshi, M. (2012) Diretrizes da International Association of Dental Traumatology para o tratamento de lesões dentárias traumáticas: Fraturas e luxações de dentes permanentes. Rev. Dent Traumatol,28, (1),2-12.

Ferrari, C. H., Simi Jr, J., \& Medeiros, J. D. (2000). Ocorrência de traumatismo dental e nível de esclarecimento e uso do protetor bucal em diferentes grupos de esportistas. Avaliable from: http://www. odontologia. com. br/artigos. asp, 1-5.

Forsberg, C. M., \& Tedestam, G. (1993). Etiological and predisposing factors related to traumatic injuries to permanent teeth. Swedish dental journal, 17(5), 183-190.

Fouad. A.F., Abbott, P.V., Tsilingaridis,, G., Cohenca, N., Lauridsen.,Bourguignon,C. ... Levin,L(2020) .Diretrizes da international association of dental traumatology para o tratamento de lesões dentais traumáticas: avulsão dos dentes permanentes. Rev.Dental Traumatology,36(6),331-342.

Granville-Garcia, A. F., Lima, E. M., Santos, P. G., \& de Menezes, V. A. (2007). Avaliação do conhecimento dos professores de educação física de CaruaruPE sobre avulsão-reimplante. Pesquisa Brasileira em Odontopediatria e Clínica Integrada, 7(1), 15-20

Gomes, M. C. B., Westphalen, V. P. D., Westphalen, F. H., Silva Neto, U. X., Fariniuk, L. F., \& Carneiro, E. (2009). Study of storage media for avulsed teeth. Brazilian Journal of Dental Traumatology, 1(2), 69-76.

Gondim, J. O., GIRO, E. M. A., Moreira Neto, J. J. S., Coldebella, C. R., Bolini, P. D. A., \& Gaspar, A. M. M. (2011). Sequelas em dentes permanentes após trauma nos predecessores decíduos e sua implicação clínica. RGO. Revista Gaúcha de Odontologia (Online), 59, 113-120.

Holan, G., Cohenca, N., Brin, I., \& Sgan-Cohen, H. (2006). An oral health promotion program for the prevention of complications following avulsion: the effect on knowledge of physical education teachers. Dental traumatology, 22(6), 323-327.

International association of dental traumatology (2020) Trauma guidelines, 2020,. Disponível em: https://www.iadt-dentaltrauma.org/. Acesso em: 22 Set. 2021.

Jorge, K. O., Ramos-Jorge, M. L., De Toledo, F. F., Alves, L. C., Paiva, S. M., \& Zarzar, P. M. (2009). Knowledge of teachers and students in physical education's faculties regarding first-aid measures for tooth avulsion and replantation. Dental Traumatology, 25(5), 494-499.

Lam, R. (2016). Epidemiology and outcomes of traumatic dental injuries: a review of the literature. Australian dental journal, 61, 4-20.

Levin, L., Samorodnitzky, G. R., Schwartz-Arad, D., \& Geiger, S. B. (2007). Dental and oral trauma during childhood and adolescence in Israel: occurrence, causes, and outcomes. Dental traumatology, 23(6), 356-359.

Lopes, L. B. P. M., \& Ferreira, J. F. (2017). Traumatismos dentários em esportes de contato. RGO-Revista Gaúcha de Odontologia, 65(3), 237-242.

Losso, E. M., dos Reis Tavares, M. C., de Paiva Bertoli, F. M., \& Baratto-Filho, F. (2011). Traumatismo dentoalveolar na dentição decídua. RSBO Revista SulBrasileira de Odontologia, 8(1), e1-e20.

Marinho, A. C. M. R., Manso, M. C., Colares, V., \& de Andrade, D. J. C. (2013). Prevalência de traumatismo dentário e fatores associados em adolescentes no concelho do Porto. Revista Portuguesa de Estomatologia, Medicina Dentária e Cirurgia Maxilofacial, 54(3), 143-149.

Menegotto, A., Scatena, C., Pereira, J. T., Werle, S. B., \& de Oliveira, R. S. (2017). Avaliação do conhecimento dos professores de escolas públicas quanto ao manejo da avulsão dentária em crianças. Revista perspectiva: ciência e saúde, 2(1), 83-94.

Mesquita, G. C., Soares, P. B. F., Moura, C. C. G., Roscoe, M. G., Paiva, S. M., \& Soares, C. J. (2017). A 12-Year Retrospective Study of Avulsion Cases in a Public Brazilian Dental Trauma Service. Brazilian dental journal, 28, 749-756.

Pacheco, L. F., Filho, P. F. G., Letra, A., Menezes, R., Villoria, G. E. M., \& Ferreira, S. M. (2003). Evaluation of the knowledge of the treatment of avulsions in elementary school teachers in Rio de Janeiro, Brazil. Dental Traumatology, 19(2), 76-78.

Poi, W. R., Sonoda, C. K., Martins, C. M., Melo, M. E., Pellizzer, E. P., Mendonça, M. R. D., \& Panzarini, S. R. (2013). Storage media for avulsed teeth: a literature review. Brazilian Dental Journal, 24, 437-445.

Rodrigues, O. F. et al (2020). Conhecimento sobre gerenciamento de avulsão de dentes permanentes em emergências entre alunos de graduação da área de saúde no Brasil. Pesqui. Bras. Odontopediatria Clín. Integr, 21(3), 327-345

Santos, A.F., Silva, N. B., Cardoso, A. M. R., \& Xavier, A. F.C. (2017). Perspectiva dos professores de educação física da rede pública sobre condutas emergenciais em avulsões dentárias. Rev. temas em saúde. 17(2),22-38. 
Research, Society and Development, v. 10, n. 14, e439101422119, 2021

(CC BY 4.0) | ISSN 2525-3409 | DOI: http://dx.doi.org/10.33448/rsd-v10i14.22119

Santos, M.F (2018). Traumatismos dentários na prática de esportes e o uso do protetor bucal. Monografia (Especialização em endododontia) - Faculdade Sete Lagoas, Vitória da Conquista, Brasil.

Silva, E. D. D., Siqueira, M. D. F. G., Gomes, M. C., Clementino, M. A., Granville-Garcia, A. F., \& Ferreira, J. M. S. (2013). Conhecimento de alunos do curso de Educação Física sobre avulsão dentária. Arquivos em Odontologia, 49(4), 177-183.

Traebert, J., Marcon, K. B., \& Lacerda, J. T. D. (2010). Prevalência de traumatismo dentário e fatores associados em escolares do município de Palhoça (SC). Ciência \& Saúde Coletiva, 15, 1849-1855.

Traebert, J., Peres, M. A., Blank, V., Böell, R. D. S., \& Pietruza, J. A. (2003). Prevalence of traumatic dental injury and associated factors among 12 -year-old school children in Florianópolis, Brazil. Dental traumatology, 19(1), 15-18.

Westphalen, V. P. D., Martins, W. D., Deonizio, M. D. A., da Silva Neto, U. X., Da Cunha, C. B., \& Fariniuk, L. F. (2007). Knowledge of general practitioners dentists about the emergency management of dental avulsion in Curitiba, Brazil. Dental Traumatology, 23(1), 6-8.

Wanderley, M. T., Coutinho, L., \& Lage-Marques, J. L (2013). Traumatismos dentários na criança e no adolescente. Odontopediatria para o Pediatra. Atheneu. 